\title{
Correction to: Sea-state contributions to sea-level variability in the European Seas
}

\author{
Antonio Bonaduce $^{1,2}$ (1) $\cdot$ Joanna Staneva ${ }^{1} \cdot$ Sebastian Grayek ${ }^{1} \cdot$ Jean-Raymond Bidlot ${ }^{3} \cdot \emptyset$ yvind Breivik ${ }^{4,5}$ \\ Published online: 9 January 2021 \\ (C) Springer-Verlag GmbH Germany, part of Springer Nature 2021
}

\section{Correction to: Ocean Dynamics (2020) 70:1547-1569 https://doi.org/10.1007/s10236-020-01404-1}

The original version of this article unfortunately contained a mistake.

In Section 6 Summary and Conclusions, "566" found in the 2nd sentence of the 7th paragraph should be omitted. The corrected paragraph should read:

The spatial signature of WIPs in the surge signals was investigated comparing 95th percentiles in the ocean-wave coupled simulations with those obtained in the reference experiments. Over a 9-year period, the largest surge values in EXP0 were observed in the German Bight $(>70 \mathrm{~cm})$ and the Baltic Sea during OND. Considering WIPs in EXP1 had a large contribution on the surge extremes ( 20\%), both over the continental shelf and in the open ocean. Sensitivity experiments performed during the year 2013 showed the contribution of WIPs over the different areas.

In addition, the legend to Fig. 13 was incorrect. The corrected legend is given below.

Fig. 13 Water-level signal. The panels show the results for EXP0 (red lines), EXP1 (blue lines) and observational records (black circles) at the Helgoland (top panel) and Husum (bottom panel) tide-gauges during December 2016. Y-axis is expressed as cm; $\mathrm{x}$-axis is expressed as days

The online version of the original article can be found at https://doi.org/ 10.1007/s10236-020-01404-1

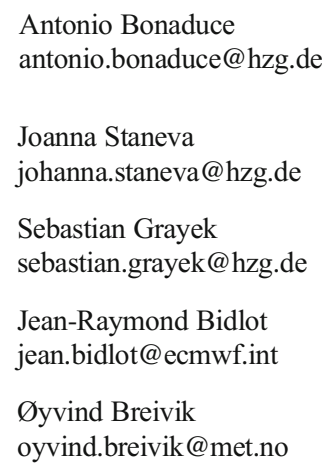

Present address: Helmholtz-Zentrum Geesthacht Centre for Materials and Coastal Research (HZG), Geesthacht, Germany

2 Nansen Environmental and Remote Sensing Center (NERSC) and Bjerknes Center for Climate Research, Bergen, Norway

3 European Centre for Medium-range Weather Forecasts (ECMWF), Reading, UK

4 Norwegian Meteorological Institute, Bergen, Norway

5 University of Bergen, Bergen, Norway 